\title{
A Study to Find out Con-Current Validity and Reliability of Mini-Balance Evaluation System Test for Assessment of Dynamic Balance in Post Stroke Patients - An Observational Study
}

\section{Desai $\mathrm{B}^{1 *}$ and Padnani $\mathrm{R}^{2}$}

${ }^{1} \mathrm{PT}$ in Neurological condition, Assistance Professor, RK University School of Physiotherapy, India

${ }^{2}$ Associate Professor at Shree K K Sheth Physiotheraphy College, India

*Corresponding author: Bhakti desai, School of Physiotherapy, RK University, B/4 bhaktidesai99@gmail.com

\section{Abstract}

Background: Stroke or brain attack is a sudden loss of neurological function caused by an interruption of the blood flow to the brain. Balance control is a fundamental motor behaviour in stance phase of gait that allows an individual to maintain and adopt various postures, react to external perturbances, and use automatic postural responses that precede voluntary movements. So purpose this study was designed to find out reliability and concurrent validity of Mini-BESTest for assessment of dynamic balance in post stroke patients.

Objective: To find out concurrent validity and reliability of Mini-BESTest for assessment of dynamic balance in post stroke patients.

Methodology: An observational study consisted of 32 post stroke patients, both male and female with age group between 30-65 years. Written consent was taken who fulfilled the selection criteria and were willing to participate in the study. After explaining whole procedure, the patients were assessed using Mini-BESTest by two rater for inter-rater reliability and by same rater at different time (24 hours of duration) for test intra-rater reliability. The patients were also assessed by Berg Balance Scale (BBS) to find out concurrent validity of Mini-BESTest.

Results: Results was analysed by using SPSS 20 version for windows software. Intra-rater and inter-rater reliability of Mini-Balance Evaluation System Test were assessed by Spearman's correlation coefficient $\left(\mathrm{r}_{\mathrm{s}}\right)$. Mini-Balance Evaluation System Test and Berg Balance Scale were assessed by Spearman's correlation coefficient. Spearman correlation 
coefficient $\left(r_{s}\right)$ for intra rater reliability is .862 with $\mathrm{p}<0.05$ and for inter rater reliability $r_{s}=.774$ with $p<0.05$. Spearman correlation coefficient $\left(r_{s}\right)$ between Mini-BESTest and BBS is 0.537 with $p<0.05$ respectively.

Conclusion: As a clinical test, Mini-BESTest is reliable and valid tool to use in post stroke patients for assessment of dynamic balance, easy to score, quick to administer (5-10 min), requires little space. Also rehabilitation can be planned according to component of score.

Keywords: Stroke; Mini-Balance Evaluation System Test (Mini-BESTest); Berg Balance Scale (BBS), Balance; Reliability and Validity

Abbreviations: BBS: Berg Balance Scale; CNS: central nervous systems; COM: Center of Mass; BOS: Base of Support; BBA: Brunal Balance Assessment; BEST: Balance Evaluation System Test.

\section{Introduction}

The term Stroke or brain attack is defined as the sudden loss of neurological function caused by an interruption of the blood flow to the brain [1]. This cut off the supply of oxygen and nutrients, causing damage to the brain tissue [2]. The most common symptom of a stroke is sudden weakness or numbness of the face, arm, or leg, most often on one side of the body, occurring in $90 \%$ of the strokes [2]. These sites generally include bifurcations, constrictions, dilation, or angulations of arteries [1]. The effects of stroke depend on which part of the brain is injured and how severely it is affected. A very severe stroke can cause sudden death [2].

Stroke is global health problem. It is second commonest cause of death and fourth leading cause of disability worldwide [3]. In developed countries, stroke is the first leading cause of disability, second leading cause of death [3]. In India, the overall prevalence rate for stroke is lies between 84 - 262 per 100,000 in rural area and between $334-424$ per 100,000 in urban areas [4]. Mean age for onset of stroke for men in India ranges from 63-65 years and for women 57-68 years [4]. Indian studies have shown that about $10-15 \%$ of strokes occur in people below age of 40 years [4].

Sensory impairments are reported in about 53\% of patients with stroke on the affected side and can range from loss of superficial and/or deep sensations and impairments in combined cortical sensations. Proprioceptive losses are also common and may lead to impairments in motor control, postural function and balance. Some patients may also suffer from central poststroke (thalamic) pain [1].

Balance is achieved by the complex integration and coordination of multiple body systems including the vestibular, visual, auditory, motor, and higher level premotor systems. Information from sensory systems is interpreted in the central nervous systems (CNS) based on an internal body schema, an appropriate response is formulated, and the postural muscle synergies are activated to perform the appropriate head, eye, trunk and limb movements to maintain posture [5].

Maintaining balance encompasses the acts of maintaining, achieving or restoring the body center of mass (COM) relative to the base of support (BOS), or more generally, within the limits of stability [6]. The functional goals of the balance system include:

1. Maintenance of a specific postural alignment, such as sitting or standing.

2. Facilitation of voluntary movement, such as the movement transitions between posture.

3. Reactions that recover equilibrium to external disturbances, such as a trip, slip, or push [5].

Balance impairments increase fall risk, resulting in high economic costs and social problems [7]. Although residual impairment is common, most people with stroke will regain walking ability; however, poor balance and impaired gait can persist [8].

After stroke, impaired dynamic standing balance can limit the ability to walk safely and increase the risk of having a fall. Because balance control is adapted to suit the task and environment, and involves many factors, clinicians examine dynamic balance using a range of clinical tests. Tests that include tasks that are relevant for daily life, such as stepping over objects and turning, and 


\section{Annals of Physiotherapy \& Occupational Therapy}

also have the potential to identify people at risk of falling, would contribute valuable information to clinical decision-making during stroke rehabilitation [9].

There are many scales to measure dynamic balance like Berg Balance Scale(BBS), Brunal Balance Assessment(BBA), Four Square Step Test(FSST) in post stroke patients. The Mini-Balance Evaluation System Test(Mini-BESTest) is a short version of the Balance Evaluation System Test(BEST). It was developed by Franchignoni and Horak's in 2010, focuses on dynamic balance, can be conducted in 10-15 min. The MiniBalance Evaluation System test is designed to access a dynamic balance assessment tool that aims to target and identify 4 different balance control systems so that specific rehabilitation approaches can be designed for different balance deficits. This test access reactive postural control, anticipatory, sensory orientation and dynamic gait. Each item is scored from 0-2. The maximum score is 28 points $[10,11]$.

Berg's Balance scale (BBS) is common tool used to assess balance in stroke patients. In 1995 Berg K et al, found the reliability of Balance scale at Center for Gerontology and Health Care Research, Brown University, USA in elderly residents with stroke. Before that in 1989, it was used with 38 balance items in the scale. In BBS, a subject is assessed with 5 point ordinal scale ranging from 0 to 4 with higher scores awarded on the basis of speed, stability or degree of assistance required for completion of the task. The task scores are summed to give a total BBS score out of a possible 56 points with higher scores representing better balance [6].

There is no such study which found out the concurrent validity and reliability of Mini-BESTest with Berg Balance Scale in post stroke patients of Indian population. So, the purpose of this study was to find concurrent validity and reliability of Mini-BESTest for assessment of dynamic balance in post stroke patients.

\section{Aim of the Study}

The aim of the study was to find out concurrent validity and reliability of Mini- Balance Evaluation System Test for assessment of dynamic balance in post stroke patients.

\section{Objectives of the Study}

1. To assess intra-rater and inter-rater reliability of the Mini-BESTest for assessment of dynamic balance in post stroke patients.
2. To assess concurrent validity of the Mini-BESTest for assessment of dynamic balance in post stroke patients.

\section{Hypothesis}

\section{Null Hypothesis}

Mini-BESTest is not having significant con-current validity, inter-rater and intra-rater reliability for assessment of dynamic balance in post stroke patients.

\section{Alternative Hypothesis}

Mini-BESTest is having significant con-current validity, inter-rater and intra-rater reliability for assessment of dynamic balance in post stroke patients.

\section{Material and Methodology}

\section{Method of collection of data}

- Study population: Post stroke patients.

- Sample size: 32 Post stroke patients.

- Sampling method: Convenient sampling.

- Study Design: An Observational study.

\section{Criteria for selection}

\section{Inclusion Criteria}

- Age: Between 30-65 years [2].

- Gender: Both male and female.

- Ambulatory post stroke patients of any stage.

- Subjects with normal cognitive function (MMSE >24) [6].

- Independently Ambulatory patient with or without use of assistive device.

\section{Exclusion Criteria}

- Musculoskeletal injuries like fractures, dislocation, joint instability or any soft tissue injuries on affected side or non-affected side.

- Severe joint disease or contracture on hemiplegic side.

- Neurological conditions other than stroke.

- Patients with severe contractures due to spasticity.

- Hemodynamically unstable patients.

After the approval of the study from the ethical committee, 32 patients from Out Patient Department (OPD) were taken, who fulfilled the inclusion and exclusion criteria taken for the study purpose. After that explanation about the purpose and procedure of this study, patients who were willing to participate in this study were requested to sign a written consent form. The selection of patients was done by 
convenient sampling. A pre-participation evaluation form consisting of basic neurological assessment chart was filled. The data measured were recorded in the measurement form which included name, age, gender, occupation, address, dominance, affected side, post stroke duration, investigation, scale total. Patients were then explained about the test and procedure to be conducted.

Mini-Balance Evaluation System Test conducted to check dynamic balance in post stroke patients. This scale divided mainly into 4 Component, Anticipatory, Reactive to postural control, Sensory orientation and Dynamic gait. These four components again divided to their subdivision. The Mini-Balance Evaluation System Test(Mini-BESTest) was conducted twice by same rater (Rater A1 and Rater A2) at different time (after 24 hours of duration) and also by different rater (Rater B) at same time to find out intra rater (test-retest) and inter rater reliability $[10,11]$.

Berg balance scale was taken to find out concurrent validity. It was 14 item scale and maximum score is 56 .

\section{Result and Table}

All the statistical analysis was done by Statistical Package for the Social Sciences (SPSS) statistical software version 20.0 for windows. Microsoft excel was used to generate graphs and tables.

Mean and standard deviation were calculated as measure of central tendency and measure of dispersion respectively. Normality of data was checked by using Kolmogorov-Smirnov test which shows that data is of non-parametric type. Intra rater and inter rater reliability of Mini-Balance Evaluation System Test were assessed by Spearman's correlation coefficient. Concurrent validity of Mini-Balance Evaluation System Test with Berg Balance Scale was assessed by Spearman's correlation coefficient. Level of significance ( $\mathrm{p}$ value) was set to 0.05 level.

The mean age of 32 post stroke patients was 55.75 years with a standard deviation of 3.53 years.

\begin{tabular}{|c|c|c|c|}
\hline Outcome Measure & Rater & Mean & Standard deviation \\
\hline \multirow{2}{*}{ Mini-Balance Evaluation System Test } & Rater A1 & 11.65 & 6.08 \\
\cline { 2 - 3 } & Rater A2 & 13 & 6.57 \\
\cline { 2 - 3 } & Rater B & 11.4 & 5.21 \\
\hline Berg Balance Scale & Rater A1 & 39.15 & 11.03 \\
\hline
\end{tabular}

Table 1: Mean value and Standard Deviation of Mini-Balance Evaluation System Test and Berg Balance Scale in post stroke patients.

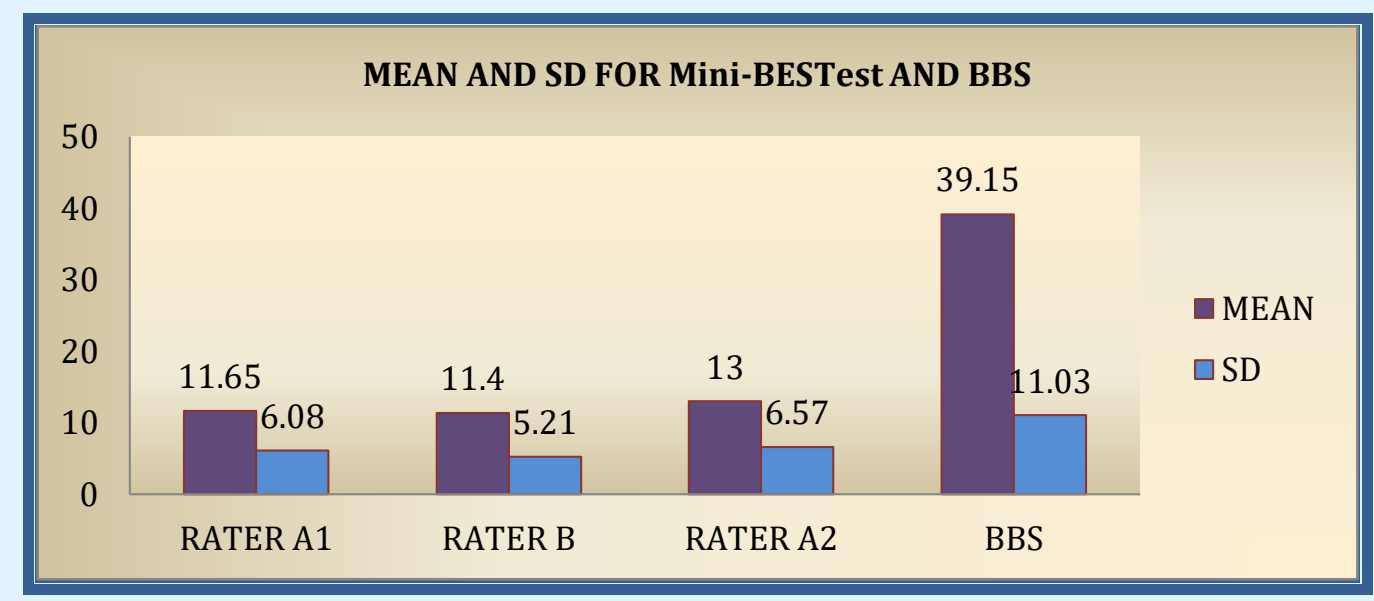

Graph 1: Mean value and Standard Deviation (SD) of Mini-Balance Evaluation System Test and Berg Balance Scale of post stroke patients.

Interpretation: Table 1 and Graph 1 are showing Mean post stroke patients. and Standard Deviation (SD) of Mini-BESTest and BBS of 


\section{Annals of Physiotherapy \& Occupational Therapy}

\begin{tabular}{|c|c|c|c|}
\hline Measure & Spearman Correlation Coefficient & p value & No. of patients \\
\hline Rater A2 (Intra rater reliability) & $.862^{* *}$ & 0 & 32 \\
\hline Rater B (Inter rater reliability) & $.774^{* *}$ & 0 & 32 \\
\hline
\end{tabular}

Table 2: Spearman correlation coefficient showing Reliability of Rater A1 of Mini-Balance Evaluation System Test with other measures.

** Correlation is significant at the 0.05 level ( $\mathrm{p}$ value $<0.05$ )

Interpretation: Spearman correlation coefficient $\left(\mathrm{r}_{\mathrm{s}}\right)$ between Rater A1 and Rater A2 is .862 with $\mathrm{p}=.000$ Spearman correlation coefficient $\left(\mathrm{r}_{\mathrm{s}}\right)$ between Rater A1 and Rater B is .774 with $\mathrm{p}=.000$. Above table shows moderately positive correlation between Rater A1 and Rater A2; Rater A1 and Rater B.

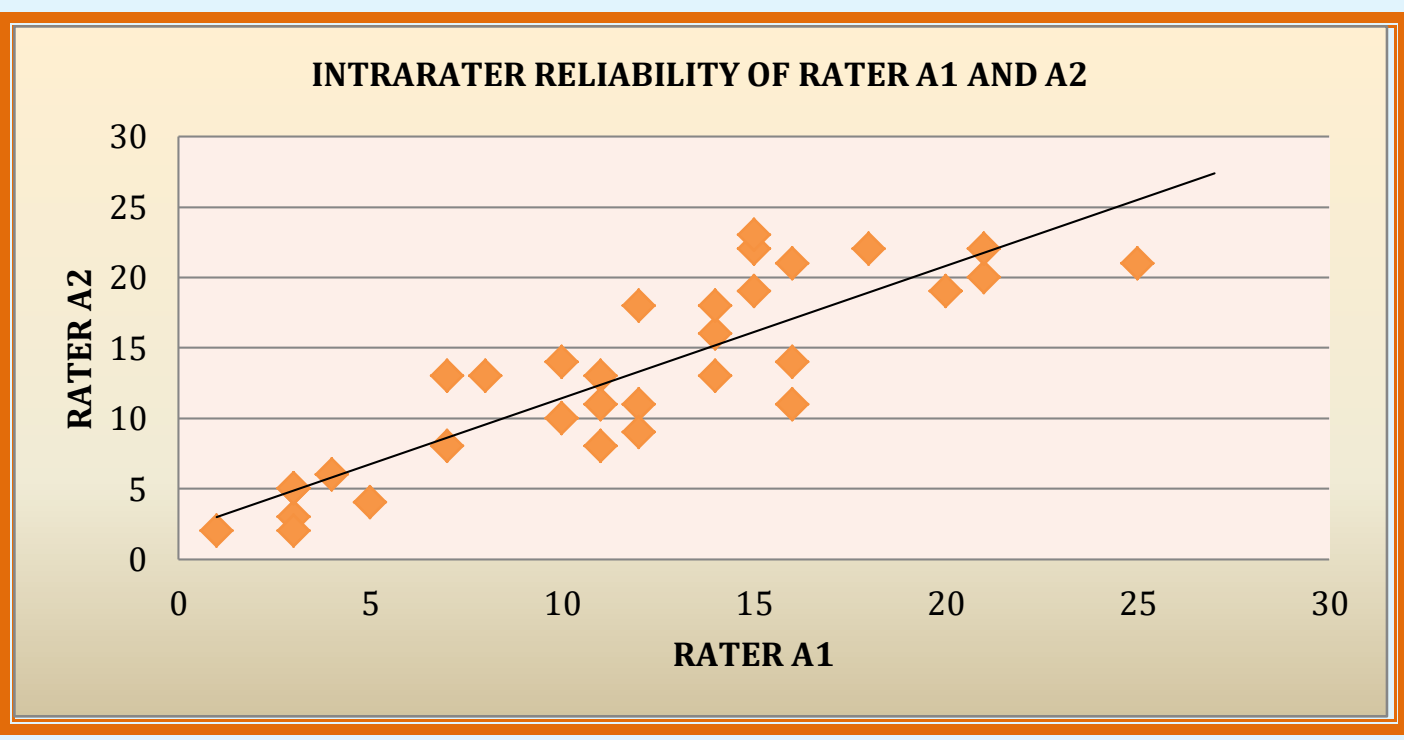

Graph 2: Result of Spearman's correlation coefficient for Intrarater Reliability of Mini-Balance Evaluation System test.

\begin{tabular}{|c|c|c|c|}
\hline Measure & Spearman Correlation Coefficient & p value & No. of patients \\
\hline Mini-BEST & $.537\left(^{* *}\right)$ & 0.002 & 32 \\
\hline BBS & $.537\left(^{* *}\right)$ & 0.002 & 32 \\
\hline
\end{tabular}

Table 3: Spearman correlation coefficient showing concurrent validity of Mini-Balance Evaluation System Test with Berg Balance Scale of post stroke patients.

** Correlation is significant at the 0.05 level ( $p$ value $<0.05$ ).

Interpretation: Spearman correlation coefficient $\left(\mathrm{r}_{\mathrm{s}}\right)$ between Mini-Balance Evaluation System Test and Berg Balance Scale is .537 with $\mathrm{p}=.002$. Above table shows moderately positive correlation between Mini-Balance Evaluation System Test and Berg Balance Scale. 


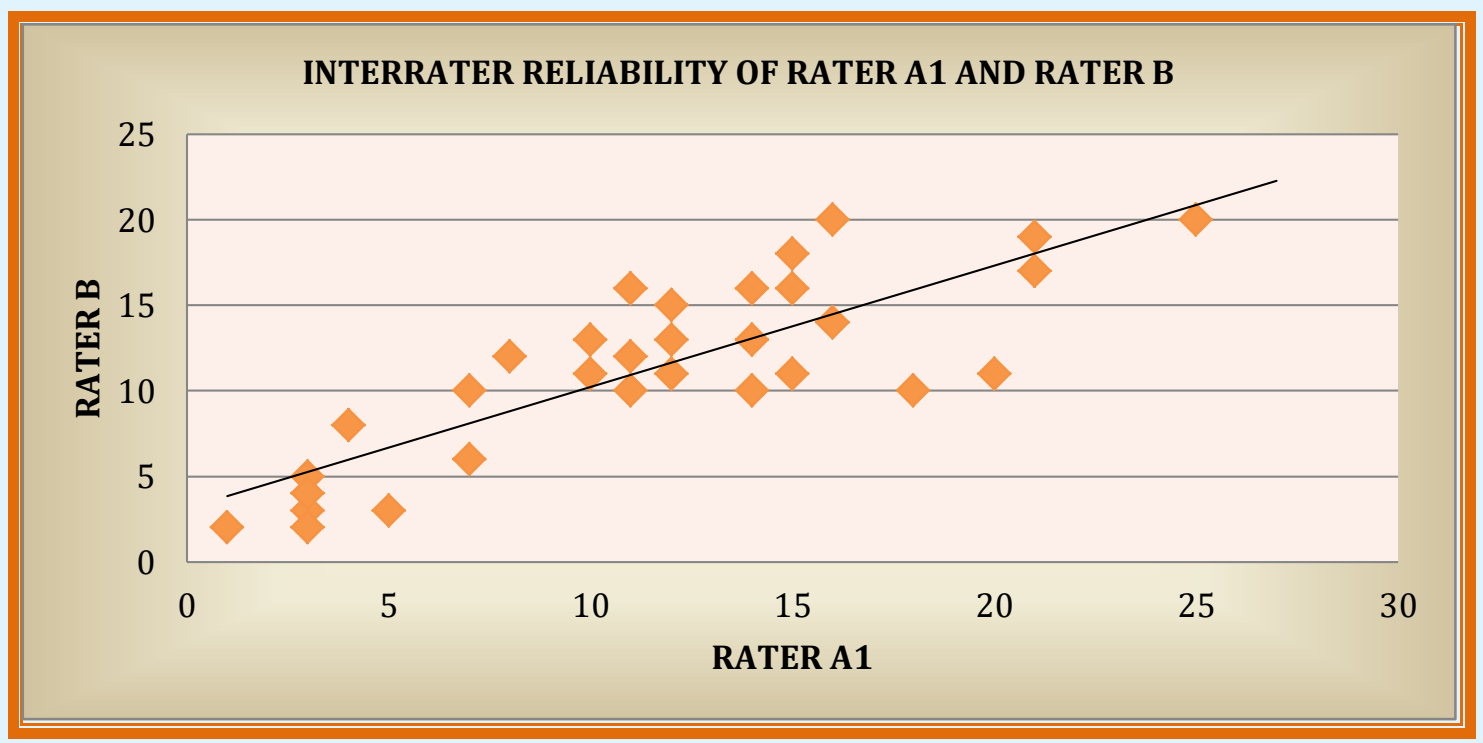

Graph 3: Result of Spearman's correlation coefficient for Interrater Reliability of Mini-Balance Evaluation System Test.

\section{Discussion}

This study was conducted to check con-current validity and reliability of Mini-Balance Evaluation System test for assessment of dynamic balance in post stroke patients.

In the above study the results for intra-rater and interrater reliability suggested moderately positive correlation with Rater A1 and Rater A2 and Rater A1 and B both which suggest that dynamic balance can reliably be measured in post stroke patients by using Mini-Balance Evaluation System Tests.

Thus, the results of the present study rejects the null hypothesis and supports the alternative hypothesis i.e. Mini-BESTest is a valid and reliable measure to assess dynamic balance in post stroke patients.

The intra-rater reliability of the Mini-BESTest is there and it would only take a clinician 1 to 2 minutes to explain, perform, and record the Mini-BESTest score and also inter rater reliability of Mini-BESTest is also there so there is no significant difference of scores between two rater also. The Mini-BESTest could be used as a quick screening tool to assess the ability to step in different directions quickly.

Balance is a complex motor skill that depends on interaction between sensorimotor processes and environmental and functional contexts. Stroke can affect different functions independently or in combination, causing heterogeneous neurological impairments and compensatory strategies. Because of such diversity, individualized rehabilitation is likely to benefit from precise assessment of each patient's impairments in motor, sensory, and cognitive aspects of postural control, as well as the functional implications. Different tools for balance assessment have been validated and should be chosen according to individual characteristics of patients with stroke [12].

Result of present study suggested that Mini-Balance Evaluation System Test is valid and reliable tool to asses balance in post stroke patients and this is supported by a study done by Charlotte SL, et al. who found out the psychometric properties of the Mini- Balance Evaluation System Test (Mini-BESTest) in chronic stroke patients and concluded that it is valid and reliable tool for evaluating balance in people with chronic stroke [7].

During the study it was observed that of Mini-BESTest performance highlighted that people with stroke who can ambulate, require more time to step safely over objects, and yet often have difficulties maintaining balance and difficulty in compensatory stepping correction forward, backward, and lateral. These difficulties are likely to have an impact on the person's capability to walk independently within the hospital, home, and community. 


\section{Annals of Physiotherapy \& Occupational Therapy}

The Berg does not include tests of postural reactions or dynamic gait, and, therefore, some deficits may be missed. Since the Mini-bestest is essentially a combination of tests, this may be a reason it successfully identified people with mild balance deficits. The Berg Balance Scale (BBS) was not designed with such system in mind but if a system categorization is assigned to each item, the berg items primarily evaluate anticipatory and sensory contribution to balance. There are two additional system that the Mini-BESTest evaluates, dynamic gait and reactive postural control. So, it can be also concluded that Mini-BESTest is also measured balance and plan a rehabilitation program according to which component score low [13].

Clinicians commonly use single-limb stance for balance assessment. An example of a difference between testing items in the BBS and Mini-BESTest is the assessment of the single-limb stance (item 14 BBS, item 3 Mini-BESTest). In the Berg, the patient chooses either leg. Comparatively, the Mini-BESTest assesses both the left and right leg and records the worst side [13].

From the present study it can be said that MiniBESTest is easy and faster to administer to measure dynamic balance this is supported by a study did by Franco Franchignoni, et al. on psychometric techniques to improve the balance evaluation system test, and found that the new 14- item scale focuses on dynamic balance, can be conducted in 10-15 minutes, and contains items belonging evenly to four of the six sections from the original BESTest. It is a unique, brief clinical rating scale for dynamic balance but further study is needed to confirm the result [11].

\section{Limitations of the Study}

1. Type and site of lesion was not considered.

2. Duration of stroke was not taken into consideration

3. Mini-Balance Evaluation System test performance might involve multiple determinants not measured in present study, such as lower limb proprioception, tactile sensation, level of spasticity, muscle strength, movement plan and level of fear of falling.

\section{Further Recommendation}

- Future research can be conducted by taking control group.

- Study can be performed to compare if there is any difference in recovery among acute, sub-acute and chronic patients.
- Physiotherapist with different years of experience can be taken to check inter rater reliability.

- Study can be done by taking different arterial inclusion e.g. Anterior Cerebral Artery where lower limb is more affected and also Posterior Cerebral Artery affection where perception and cognition is likely to be altered.

- Study can also be done by taking different outcomes in relation with balance like, proprioception, sensation etc.

\section{Conclusion}

The Mini-BESTest appears to be reliable and valid test to examine how people with stroke affect movement to walk over obstacles, anticipatory control, dynamic gait and reactive postural control in various directions. The Mini-BESTest is an easy-to-administer, less time consuming clinical test with concurrent validity, intarrater and inter-rater reliability for assessment of dynamic balance in post stroke patients.

Acknowledgement: I would like to thanks Dr. Ashish Kakkad and Dr. Paras Joshi for guidance. Also my parents $\&$ friends for their support

Conflict of Interest: There was no personal or institutional conflict of interest for this study.

Ethical Clearance: From K.K. Sheth Physiotherapy College, Rajkot.

\section{References}

1. Susan B O Sullivan, Thomas J Schmitz (2007) Physical rehabilitation. $5^{\text {th }}$ (Eds.), Jaypee Brothers.

2. Manjari Tripathi, Deepti Vibha (2010) Review Article Stroke in Young India. Hindawi Access to Research. Stroke Research and Treatment.

3. Go AS, Mozaffarian D, Roger VL, Benjamin EJ, Berry JD, et al. (2014) Heart disease and stroke statistics-2014 update: a report from the American Heart Association. Circulation 129(3): e28-e292.

4. Jeyaraj Durai Pandian, Paulin Sudhanb (2013) Stroke Epidemiology and Stroke Care Services in India. J Stroke 15(3): 128-134.

5. Martina Mancini, Fay B Horak (2010) The relevance of clinical balance assessment tools to differentiate balance deficits. Eur J Phys Rehabil 46(2) 239-248. 


\section{Annals of Physiotherapy \& Occupational Therapy}

6. Katherine Salter, Nerissa Campbell, Marina Richardson, Swati Mehta, Jeffrey Jutai, et al. (2013) Outcome Measures in Stroke Rehabilitation. Chapter: 21.

7. Tsang CS, Liao LR, Chung RC, Pang MY (2013) Psychometric properties of the Mini-Balance Evaluation System Test (Mini-BESTest) in community- dwelling individual with chronic stroke. Phys Ther 93(8): 1102-1115.

8. Carolyn Kisner, Lynn Allen Colby (2007) Theraputic exercise: Foundation \& technique. $5^{\text {th }}$ (Eds.).

9. Whitney S, Marchetti G, Morris L, Sparto P (2007) The reliability and validity of the Four Square Step test for people with balance deficits secondary to a vestibular disorder. Arch Phys Med Rehabil 88(1): 99-104.
10. Rehab measures: Mini Balance Evaluation System Test.

11. Franchignoni F, Horak F, Godi M, Nardone A, Giordano A, et al. (2010) Using psychometric techniques to improve the Balance Evaluation System's Test: the mini-BESTest; J Rehabil Med 42(4): 323-331.

12. de Oliveira CB, de Medeiros IR, Frota NA, Greters ME, Conforto AB, et al. (2008) Balance control in hemiparetic stroke patients: main tools for evaluation. J Rehabil Res Dev 45(8): 1215-1226.

13. King LA, Priest KC, Salarian A, Pierce D, Horak FB, et al. (2012) Comparing the Mini-BESTest with the Berg Balance Scale to Evaluate Balance Disorders in Parkinson's Disease. Parkinsons Dis 375419. 